\title{
Auf dem Weg zur Normalität: Planungsbezogener Protest und planerische Reaktionen
}

\section{Towards Normality: Planning-related Protests and Reactions by Planning}

https://doi.org/10.2478/rara-2019-0059

Eingegangen: 11. Februar 2019; Angenommen: 14. Oktober 2019

\begin{abstract}
Kurzfassung: Der Beitrag plädiert für eine stärkere Unterscheidung von Planungsprotesten, die Teil der täglichen Planungspraxis sind. Es wird argumentiert, dass die Unterschiedlichkeit auch für differenzierte Reaktionen der Planungsakteure genutzt werden kann. Skizziert wird der Forschungsstand sozialwissenschaftlicher Protest- und Bewegungsforschung sowie die Besonderheit planungsbezogenen politischen Protests. Planungsprotest wird dabei als kollektives Handeln und Form kommunalpolitischer Partizipation verstanden, die unabhängig von lokalstaatlichen Beteiligungsangeboten stattfindet und einen Bezug zur lokalen räumlichen Planung aufweist. Basierend auf einer empirischen Erhebung und statistischen Untersuchung von mehr als 400 planungsbezogenen Bürgerprotesten in Berlin zwischen 2005 und 2015, die durch zwei qualitative Fallstudien ergänzt werden, wird eine - orts- und zeitspezifische -Typologie von neun Protestarten entwickelt. Die im Framing der Protestakteure artikulierten Protestanlässe und Protestanliegen werden als alternative Problemlösungsansätze verstanden. Unterschieden werden situationsbezogene und planungsabhängige sowie initiative und reaktive Protestformen. Diese inhaltliche Unterscheidung erscheint zwar in besonderem Maße für das aktuelle Protestgeschehen von Bedeutung und unterstreicht dessen politischen Charakter, sie ist allerdings nur ein Teil der phänomenologischen Vielfalt. Auch die Reaktionen sind fallspezifisch zu unterscheiden, wie das Merkmal der Betroffenheit bzw. Nicht-Betroffenheit der Protestakteure verdeutlicht.
\end{abstract}

Schlüsselwörter: Protest, Partizipation, Planungsinhalte, Typologie, Berlin

\begin{abstract}
The paper argues for a stronger distinction within planning protests, which is today an everyday part of planning practice. Furthermore, it is argued that the diversity of planning protest can also be used for differentiated reactions of planning practitioners. It is possible and sensible to adopt theories from protest and social movements research to better understand protest in planning, yet specifications for planning-related protest are needed. Planning protests are understood as collective action and form of local political participation, which takes place independently participation offers and has a relation to local spatial planning. Based on an empirical survey and statistical analysis of more than four hundred planning-related citizen protests in Berlin between 2005 and 2015, supported by two qualitative case studies, a typology of nine protest types will be developed, that is specific in time and place. The causes and claims articulated in the framing of protesters are understood as alternative approaches to solving planning problems. In particular, a distinction is made between situational and planning-dependent as well as initiative
\end{abstract}

\footnotetext{
*Corresponding author: Dr. Grischa Frederik Bertram, Universität Kassel, Stadterneuerung und Planungstheorie, Gottschalkstraße 22, 34127 Kassel, Deutschland, E-Mail: grischa.bertram@uni-kassel.de

Prof. Dr. Uwe Altrock, Universität Kassel, Stadterneuerung und Planungstheorie, Gottschalkstraße 22, 34127 Kassel, Deutschland
} 
and reactive types of protest. Differences in content appear to be of special importance for the current protests, but also underline its political character. Yet, they are only part of the phenomenological diversity of planning protest and reactions therefore are to be differentiated for every individual case. This will be highlighted with regard to protests that are organised either by affected or unaffected citizens.

Keywords: Protest, Participation, Planning policy, Typology, Berlin

\section{Planungsprotest: Eine Einführung}

Planungsprotest scheint derzeit in Planungspraxis und Fachdebatten allgegenwärtig. Spätestens, seitdem mit „Stuttgart 21“ ein lokaler planungsbezogener Protest auch bundesweit Schlagzeilen machte, sind Bürgerproteste und ihre (vermeintliche) Zunahme ein häufiges Thema. Dabei scheint der Stuttgarter Fall kaum verallgemeinerbar. Gibt es überhaupt so etwas wie typische Planungsproteste? Oder verschiedene Arten? Planungstheorie und Planungsforschung sind bislang kaum in der Lage, hierauf Antworten zu geben (vgl. aber erste Ansätze bei Othengrafen/Sondermann 2015), fehlt innen doch schon ein Protestbegriff, der nicht allein der Umgangssprache entlehnt ist. Dies ist insofern verwunderlich, als es nicht nur erhebliche Unterschiede zwischen dem Selbstverständnis der Protestierenden und der Zuschreibung durch Planende gibt, sondern auch innerhalb der Planung selbst: Die einen sehen Protestierende als "Störenfriede" (Selle 2011a: 1) an und Protest in der Planung wird als Protest gegen Planung verstanden, der das normale Planungsverfahren durch irrationales und affektives Verhalten störe und zu einem „Ausnahmezustand" führe (Selle 2013: 24). Bei anderen besteht eine empathische Fürsprache, die nicht zuletzt aus der Entzauberung des rationalen Planungsmodells durch Protest herrührt, in dessen Folge in der Disziplin selbst ein Paradigmenwechsel stattgefunden hat (Holm/Kuhn 2010: 107; Haughton/Gilchrist/Swyngedouw 2016: 486). Überraschenderweise führt beides bislang nicht zu einer tiefergehenden Analyse, und es besteht im Wesentlichen Einigkeit darüber, dass bessere Planungsverfahren die beste Antwort auf Planungsprotest sind (Krau 2011): entweder weil so die Fehlinterpretationen der Protestierenden durch bessere Kommunikation aufgedeckt oder weil Fehler in der Planung korrigiert werden könnten (Lopes de Souza 2006: 338; Selle 2011b: 2).

Im vorliegenden Beitrag wird untersucht, ob ein analytischer Begriff von Planungsprotest eine planungstheoretische Unterscheidung ermöglicht und einen Ansatzpunkt für einen angemesseneren Umgang in der
Planungspraxis darstellen kann. Im zweiten Kapitel wird gezeigt, dass ein solcher analytischer Begriff, der weder zu stark vereinfacht noch normativ ist, auf der Grundlage der sozialwissenschaftlichen Protest- und Bewegungsforschung zunächst zu zwei Perspektivwechseln führt: Einerseits wird Planungsprotest als normaler Bestandteil stadtpolitischer Prozesse angesehen und andererseits werden für seine Analyse nicht die Instrumente und Verfahren, sondern Planungs- bzw. Protestinhalte in den Mittelpunkt gestellt. Im dritten Kapitel wird diskutiert, inwiefern sich ein eigenständiger planungswissenschaftlicher Protestbegriff von sozialwissenschaftlichen Ansätzen emanzipieren muss. Im vierten Kapitel werden empirische Daten zu planungsbezogenem Bürgerprotest in Berlin, die im Rahmen des DFG-geförderten Forschungsprojekts „Muezzine, Fluglärm, Touristification \& mehr" erhoben worden sind, beispielhaft für eine analytische Unterscheidung unterschiedlicher Protestarten genutzt, die auch Möglichkeiten für eine Generalisierung erkennen lassen. Anschließend wird der Frage nachgegangen, ob eine solche Typologie auch einen differenzierten planungspraktischen Umgang mit Protesten ermöglicht (vgl. Kapitel 5). Im sechsten Kapitel wird diskutiert, inwiefern Planungsprotest als Normalität Teil von Demokratisierungsbemühungen in der lokalen räumlichen Planung sein kann.

\section{Politischer Protest in der Protest- und Bewegungsforschung}

In den Sozialwissenschaften wird Protest als kollektives politisches Handeln und als Form politischer Partizipation unabhängig von Wahlen und weiteren kommunalen und staatlichen Beteiligungsangeboten verstanden (Rucht 2001; vgl. auch Bertram/Altrock 2017). Demnach führen rationale Entscheidungen zu Protest und nicht kollektives Verhalten (Melucci 1985; Tarrow 2011). Bis zu einem Paradigmenwechsel ab Ende der 1960er-Jahre wurden Proteste als emergente Phänomene innerhalb 
weitgehend ungesteuerter Massen angesehen, die affekthaft auf Strukturbrüche reagieren, und ihr Auftreten sozialpsychologisch erklärt (Tarrow 2011: 22). Heute hingegen gelten Proteste als normale Ausdrucksform gut informierter, kompetenter und mit den notwendigen Ressourcen ausgestatteter Bürgerinnen und Bürger, die Protestnetzwerke gründen. Damit folgt die Analyse letztlich der Forschung zu den seit den 1970er-Jahren vielfach untersuchten so genannten Bürgerinitiativen (vgl. etwa Eckert 1970; Schiller 1970; Guggenberger 2009). Kern der Definitionsansätze ist die Vorstellung von anlassbezogenen Zusammenschlüssen, die sich weitgehend ad hoc, aber eben nicht „spontan“, während der sozialen Konstruktion des Protests gründen (Roth/Rucht 2008: 17) und sich „um Abhilfe im Sinne ihres Anliegens bemühen" (Guggenberger 2009: 40).

Der Begriff "Initiative" hebt aufgrund des häufig reaktiven Charakters für Guggenberger (2009: 39) eher auf „das Moment des unmittelbaren Tätigwerdens des Bürgers" und nicht auf Inhalte ab, doch wird darin auch eine "doppelte Signatur" des Protests deutlich, auf die vor allem Rucht (2001: 9) hinweist: Protestierende richten sich nicht nur gegen eine als Herausforderung wahrgenommene Situation, Norm oder Planung, sondern treten zugleich für eine Änderung ein und fordern gegebenenfalls sogar die Einleitung eines Planungsverfahrens. Hierin wird ein politischer Charakter des Planungsprotests deutlich (vgl. Kapitel 5.1): Protest wird in der sozialwissenschaftlichen Literatur aufgrund des Willens zur gesellschaftlichen Veränderung und wegen seines kollektiven Charakters als politisch betrachtet (Goodwin/Jasper 2003: 3; Tarrow 2011: 7). Gamson (1975: 14, 143) hebt hervor, dass daraus eine ähnliche Motivationsvielfalt resultiert wie für andere politische Strategien: Ziele können Einfluss, Mobilisierung von Anhängerinnen und Anhängern sowie (politischer) Nutzen sein. Erfolgreicher Protest kann dazu führen, dass Protestierende Teil der politischen Elite werden.

\section{Die Besonderheit planungsbezogenen politischen Protests}

Die diesem Beitrag zugrunde liegenden empirischen Untersuchungen zeigen, dass der wertfreie sozialwissenschaftliche Protestbegriff planungswissenschaftlich adaptiert werden kann. Zugleich erscheint es erforderlich, inn planungsbezogen zu spezifizieren - gerade auch in Abgrenzung zu vielfältigen Angeboten zu ,städtischen' Bewegungen, wie sie ausgehend von Castells
(1977) mittlerweile durch eine Vielzahl von Autoren und Autorinnen bestehen (etwa Mayer 2008; Künkel/Mayer 2012; Vogelpohl/Vollmer/Vittu et al. 2017; vgl. dazu kritisch Bertram 2013).

So wurde bereits im Forschungsprojekt zunächst eine Begrenzung auf lokal- bzw. stadtpolitischen Protest vorgenommen. Planungsproteste sind lokalpolitisch, wenn sie im Sinne von Fainstein und Fainstein (1985: 189) „[are] rooted in collectivities with a communal base and/or with the local state as their target of action". Nicht berücksichtigt wurden insbesondere solche Proteste, die den Stadtraum als „Bühne“ (Lindner 1996: 414) nutzten, aber auf überlokale Politiken bezogen waren. Auf eine Unterscheidung zwischen Kommunal- und Landespolitik wurde in diesem Beitrag verzichtet.

Proteste werden in diesem Beitrag als planungsbezogen betrachtet, wenn dieser Bezug innerhalb des sozialen Konstruktionsprozesses der Protestformation hergestellt wird. Dabei wurde maßgeblich das strategische Framing der Protestakteure zugrunde gelegt, eine Mobilisierungsstrategie von Protestakteuren zur Verstärkung und Erweiterung von Anlässen, Anliegen und Motivationen (Snow/Benford 1988). Entsprechend können Aussagen von Protestakteuren genutzt werden, um zu prüfen, ob sie ihre Anlässe und/oder Anliegen im Bereich der lokalen räumlichen Planung sehen: Werden Planungsinhalte oder Planungsverfahren als Herausforderung bzw. Problem beschrieben, weil der Konflikt verursacht, verstärkt oder nicht zufriedenstellend bearbeitet wird, wird die lokale räumliche Planung zum Protestanlass. Werden Planungsinhalte oder Planungsverfahren von den Protestierenden als (Teil der) Problem- bzw. Konfliktlösungen gefordert, wird sie zum Protestanliegen. Unabhängig vom Framing wird der Bezug hergestellt, indem Planungsinstitutionen direkt oder indirekt adressiert werden, innen also von Protestierenden, den Protestobjekten oder Dritten eine Rolle im Konflikt zugeschrieben wird.

Angesichts der divergierenden Auffassung darüber, wo die Grenzen der „Rubrik ,Planung““ (Lennon 2017: 147; eigene Übersetzung) verlaufen, wurde der Bezug graduell in vier Stufen unterschieden: Werden Verfahren und Inhalte der lokalen räumlichen Planung einschließlich gemeindlicher/städtischer sektoraler Planungen mit Bodenrecht direkt benannt, ist der Bezug am größten, etwa dort, wo Planwerke oder Flächennutzungen kritisiert werden. Werden hingegen nur ,Beiträge zur räumlichen Entwicklung' stadtpolitischer Akteure benannt, wird von einem losen Planungsbezug ausgegangen. Hierunter fallen etwa viele mietenpolitische Proteste, die in der Erhebung dokumentiert wurden. Proteste mit nur losem 
Planungsbezug werden im Beitrag nicht berücksichtigt. Aufgenommen wurde jedoch ein breites Spektrum von Protesten mit Bezug zu Verfahren, Maßnahmen und Vorhaben, die der kommunalen räumlichen Planung nach- oder übergeordnet sind, sowie zu Inhalten, die (potenziell) Teil raumbezogener Stadt(teil)entwicklungspläne sind.

\section{Zur Vielfalt des Planungsprotests am Beispiel der Protestinhalte}

\subsection{Methodischer Zugang und empirischer Hintergrund}

Wendet man die im vorherigen Kapitel skizzierte Definition auf die empirische Erhebung an, wird deutlich, wie alltäglich und vielgestaltig Planungsproteste inzwischen geworden sind. Um sie angemessen in die planungswissenschaftliche Prozessforschung einordnen zu können, ist eine Typologisierung erforderlich.

Den empirischen Hintergrund des Beitrags bildet das DFG-geförderte Forschungsprojekt „Muezzine, Fluglärm, Touristification \& mehr - Vorstudie zu aktuellem bürgerschaftlichen Protest in der lokalen räumlichen Planung in Berlin“, in dessen Rahmen für den Zeitraum von 2005 bis 2015416 Planungsproteste in Berlin identifiziert und näher untersucht worden sind (vgl. Bertram 2019). Der Beginn des Untersuchungszeitraums wurde so gewählt, dass damit einige Jahre vor einer vermuteten Wachstumsphase zwischen 2008 und 2011 abgedeckt waren. Da die Erhebung in den Jahren 2015 und 2016 stattfand, konnten spätere Protestereignisse nicht erfasst werden. Grundlegende Erhebungsmethode war die Protestereignisanalyse, die im Rahmen der bundesweiten Längsschnitterhebung PRODAT ${ }^{1}$ entwickelt und erprobt worden war, sich allerdings auf eine strukturierte Inhaltsanalyse von Zeitungen beschränkte (vgl. Rucht/Ohlemacher 1992). Im genannten Projekt wurde das inhaltsanalytische Verfahren deshalb ergänzend auf Internetseiten, soziale Medien und Polizeidaten zu politisch motivierter Kriminalität ausgeweitet. Zusätz-

1 Das Forschungsprojekt „Dokumentation und Analyse von Protestereignissen in der Bundesrepublik" (PRODAT) wurde zwischen 1993 und 2007 am Wissenschaftszentrum Berlin (WZB) durchgeführt. Dabei wurde insbesondere eine lange Zeitreihe politischer Proteste für die Jahre 1950 bis 2002 erstellt. lich wurden über leitfadengestützte Experteninterviews und Beobachtungen städtischer Räume weitere Datenquellen erschlossen. Dieser Methodenmix diente dazu, die Wahrnehmungsschwelle von Tageszeitungen zu überwinden und Proteste mit geringerer Öffentlichkeitswirkung einzubeziehen. Sämtliche hinreichend dokumentierten, planungsbezogenen und stadtpolitischen Protestereignisse (im Sinne der Operationalisierung in Kapitel 3) zwischen 2005 und Juni 2015 und einem Anlassort innerhalb der Berliner Landesgrenze wurden in einer Datenbank erfasst und mit 31 Merkmalen zur Vereinheitlichung der Daten und statistischen Analysen mittels PSPP, einer Software zur Analyse statistischer Daten, verschränkt (Häufigkeiten, Erwartungswerte, Kreuztabellierung). Zwei Protestereignisse wurden in qualitativen Fallstudien in Bezug auf Verlauf, Akteurhandeln und Voraussetzungen vertieft untersucht.

Als ein erstes Ergebnis bestätigt die Erhebung die Annahme, dass es in den letzten Jahren zu einem Anstieg der Zahl bürgerschaftlicher lokaler Planungsproteste gekommen ist (vgl. Walter 2013). Greift man aus dem Untersuchungszeitraum nur die Jahre 2007 bis 2013 heraus, in denen ein Protest mit einer durchschnittlichen Dauer von gut zweieinhalb Jahren von Anfang bis Ende erfasst werden konnte und somit eine valide Aussage zur Fallzahlentwicklung möglich ist, so hat sich die Anzahl in diesem Zeitraum beinahe verdoppelt. Auch wenn man davon ausgeht, dass die Fallzahlen der Internetanalyse schon aufgrund der Bedeutungszunahme des Mediums und wegen der Löschung von Internetseiten ansteigen müssen und die Werte entsprechend rechnerisch korrigiert werden, bleibt es bei einem deutlichen Anstieg der Fallzahlen. Abbildung 1 zeigt, wie viele Proteste in den Jahren 2007 bis 2013 begonnen und beendet wurden (bzw. für die erst- bzw. letztmalig Protestereignisse registriert wurden) sowie die Summe aller zum jeweiligen Zeitpunkt begonnenen, aber noch nicht beendeten Proteste (auch bei einem Beginn vor 2007 oder einer Phase der Latenz).

Erstaunlich ist die Anzahl der Teilnehmerinnen und Teilnehmer: Unabhängig vom jeweiligen Umfang der Protestaktivität haben sich zwischen Januar 2005 und Juni 2015 mindestens 2,6 Mio. Menschen nachweislich an Planungsprotesten in Berlin beteiligt. ${ }^{2}$ Dem stehen im

2 Zur Ermittlung dieser Zahl wurde die höchste in der Datengrundlage genannte Teilnehmerzahl eines Planungsprotests festgehalten und für alle Proteste addiert. Relativierend muss hier eingeräumt werden, dass auch die mehr als 700.000 Teilnehmenden am Bürgerbegehren zum Tempelhofer Feld in die Gesamtzahl eingingen und viele Protestierende vermutlich mehr als einmal gezählt wurden. Dem steht gegenüber, dass bei 


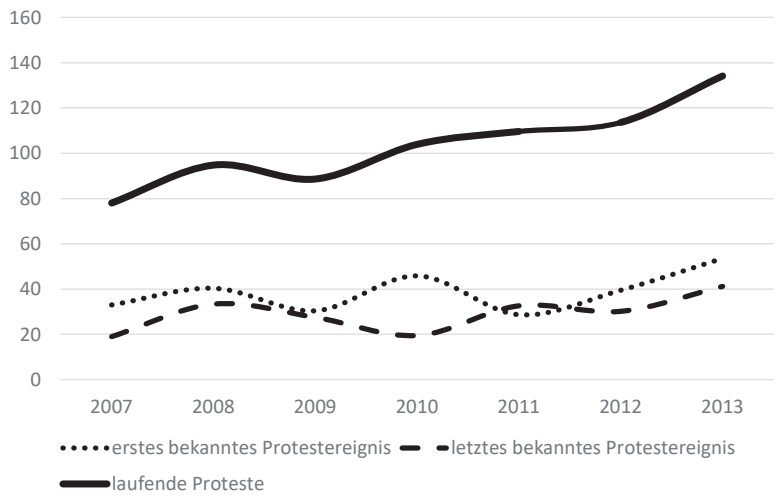

Abbildung 1: Fallzahlentwicklung für laufende Planungsproteste, erste und letzte Protestereignisse in Berlin zwischen 2007 und 2013 (korrigierte Werte; $\mathrm{N}=416$ )

Untersuchungszeitraum insgesamt 5,4 Mio. Menschen gegenüber, die irgendwann in Berlin gemeldet waren ${ }^{3}$ und bei drei Abgeordnetenhauswahlen insgesamt 2,9 Mio. Zweitstimmen abgegeben haben. Politischen Parteien gehörten als Mitglieder zirka 74.000 Menschen an. Im Durchschnitt dauerten die identifizierten Proteste 2,7 Jahre, sodass im Mittel jeweils 107 gleichzeitig liefen bei maximal 190 zu einem Zeitpunkt laufenden Protesten im Jahr 2014.

Planungsproteste können insofern mit Fug und Recht als alltägliche Ereignisse betrachtet werden. Dies ist nicht allein als Abgrenzung zu umfassenderen Protesten auf übergeordneten politischen Ebenen anzusehen (vgl. Haughton/Gilchrist/Swyngedouw 2016). Vielmehr zeigt sich darin ihre Selbstverständlichkeit und schiere Häufigkeit. Man kann vermuten, dass dieser Befund der besonderen politischen Möglichkeitsstruktur in Berlin geschuldet ist - (West-)Berlin ist nicht nur als Hochburg der Studierendenbewegung und alternativer Bewegungen bekannt geworden. Den 15 bis 25 Jahre nach der deutschen Wiedervereinigung liegenden Untersuchungszeitraum prägten die Folgen eines massiven sozioökonomischen Transformationsprozesses, der vielerorts zur Notwendigkeit geführt hat, die Produktion städtischer Räume neu zu verhandeln. Dabei ging es nach dem Abbau der Berlin-Subventionen und der Wiedergewinnung der Hauptstadtfunktion um Entwicklungen von der Wiedernutzung vormals brachgefallener Flächen bis zum Umgang mit einem inzwischen beträchtlichen Bevölkerungswachstum im Umfeld einer

vielen Protesten gar keine Teilnehmerzahl bekannt ist, die in die Ermittlung der Gesamtzahl hätte eingehen können.

3 Zugleich waren maximal 3,7 Mio. Menschen zu einem Zeitpunkt gleichzeitig in Berlin ansässig. aufstrebenden Dienstleistungsökonomie (vgl. Brake 2012). Die wachsende Bevölkerung traf und trifft unter anderem auf ehemalige Hausbesetzer und Hausbesetzerinnen, ein kreatives Prekariat sowie ehemalige OstBerliner Regimekritiker und Regimekritikerinnen, sodass gute Ausgangsbedingungen beispielsweise für einen Protest eher kapitalismuskritischer Milieus gegen global agierende Investoren bestehen.

Überregionale Aufmerksamkeit und lokalen Widerstand hat etwa der Vorhabenkomplex "Mediaspree" zur Überformung des Friedrichshain-Kreuzberger Spreeufers durch international agierende Medienkonzerne ausgelöst (vgl. Dohnke 2014). Die Auseinandersetzung um die Zukunft des teilweise öffentlich zugänglichen Uferbereichs und dessen befürchtete Privatisierung kulminierte 2008 in einem Bürgerentscheid, der Investoren und Senat mindestens zu einem Strategiewechsel bei deren Durchsetzung zwang.

Doch man sollte sich nicht täuschen: Vielfach handelt es sich bei den Protesten gar nicht um den Ausdruck von Widerstand gegen spektakuläre Übernahmen kreativ zwischengenutzter innerstädtischer Flächen durch Akteure eines global vernetzten Kapitalismus. Im wohl bekanntesten Fall, dem Protest gegen eine Teilbebauung des ehemaligen Tempelhofer Flugfelds in WestBerlin, trauten die Gegnerinnen und Gegner gerade dem lange Zeit beliebten Senat aus einer Koalition von SPD und Die Linke unter Klaus Wowereit nicht mehr. Er hatte unter anderem versprochen, landeseigene Wohnungsgesellschaften mit der Realisierung von Sozialwohnungen $\mathrm{zu}$ betrauen und verstreut angesiedelte Bibliotheksstandorte zu vereinen. Zumeist stellen sich die Proteste weniger spektakulär und oft gewissermaBen ,bürgerlicher' dar als im Beispiel „Mediaspree“. Drohende Mietsteigerungen durch Sanierungsmaßnahmen gehören genauso dazu wie die Bebauung von Baulücken, die als wichtige kleine Freiflächen in einer dicht bebauten Innenstadt betrachtet werden. Die Fällung von Bäumen, der Abriss von Gebäuden, die Einführung einer Parkraumbewirtschaftung oder die Erhebung von Straßenausbaubeiträgen werden genauso zum Gegenstand von Protesten wie der Unmut über Bahnlärm oder das Fehlen eines Zebrastreifens. Die Zahlen der Beteiligten und die Aktionsformen deuten darauf hin, dass Proteste mit eindeutigerem Planungsbezug tendenziell umfangreicher und in gewisser Weise auch ,spektakulärer' sind.

Besonders augenfällig bringen die zwei im DFGProjekt in Fallstudien vertieft untersuchten Vorhaben auf den Punkt, dass sich Planungsproteste in Berlin pauschalen Darstellungen entziehen. Das erste Vorhaben, im Südosten Berlins, betrifft die Planung einer größe- 
ren überbezirklichen Hauptverkehrsstraße im Umfeld eines weitgehend durch Einfamilienhäuser geprägten Stadtteils. Entgegen der Vermutung, dass die Sorge vor zusätzlichem Verkehr und dessen Auswirkungen eine prinzipielle Ablehnung vor Ort hätte auslösen können, ging es dort aber gar nicht um die grundsätzliche Sinnhaftigkeit der Straße. Vielmehr setzten sich die Protestierenden für die Umsetzung einer abgeänderten Planungsalternative ein, die vor Ort im Planungsprozess durchaus in Betracht gezogen worden war. Die Protestierenden erreichten mit ihrer konstruktiven Mitwirkung eine Neubewertung der Alternativen, die letztlich zu einer veränderten Einschätzung der jeweils zu erwartenden Auswirkungen führte.

Das zweite Vorhaben drehte sich um die Umwandlung einer innenstadtnahen Kleingartenfläche in der westlichen Innenstadt in ein Wohnquartier. Möglich geworden ist sie nicht etwa deswegen, weil in Zeiten einer entfesselten Wohnungsnachfrage die städtische Politik auf eine forcierte Überbauung drängte. Vielmehr versuchte der Bezirk über lange Zeit vergeblich, die Fläche als Kleingartenfläche zu sichern. Er scheiterte aber, als nach einem Grundstücksgeschäft Entschädigungsansprüche durch den neuen Eigentümer aufgrund der rechtlich umstrittenen Tragweite eines historischen, aber gleichwohl weiterhin gültigen Plans befürchtet wurden. Der anfängliche Kleingärtnerprotest gipfelte schließlich in einem Bürgerentscheid, der weit über die lokalen Akteure hinaus Zustimmung fand und mit dem Willen der bezirklichen Politik übereinstimmte.

Schon diese Einblicke machen deutlich, wie komplex die Protestwirklichkeit in Berlin ist. Die im Folgenden dargestellten empirischen Befunde sollen im Zusammenhang mit dem Versuch einer Protest-Typologie einen ersten Schritt auf dem Weg zu einem solchen vertieften Verständnis ermöglichen.

\subsection{Planungsinhalte als zentraler Anlass für Planungsproteste}

In der deutschsprachigen Literatur entwickelten Othengrafen und Sondermann (2015: 12 f.) eine Einteilung nach Protestursachen mit vier sich gegebenenfalls überlagernden Konfliktarten (Ziel-, Werte-, Mittel- sowie strukturelle Konflikte). Planungsproteste seien stets Betroffenenproteste. In ähnlicher Weise sind es auch an anderer Stelle eher Abgrenzungen einzelner phänomenologisch begründeter Protestarten, die nur ansatzweise zutreffend charakterisiert werden. Das trifft insbesondere auf die in der internationalen Literatur häufig benannten
NIMBY-Proteste ${ }^{4} \mathrm{zu}$, denen aufgrund einer (vermeintlichen) Abwehrhaltung eine geringe Gemeinwohlorientierung unterstellt wird (vgl. dazu kritisch Lake 1993). In der angloamerikanischen Literatur werden dem Begriff weitere Akronyme zur Seite gestellt (vgl. Menzl 2014), sodass hier so etwas wie eine Typologie ablehnender Fremdzuschreibungen entsteht. Da Planungsproteste häufig gemeinsam mit städtischen Bewegungen betrachtet werden, gibt es in der entsprechenden Literatur eine Vielzahl von Unterscheidungs- bzw. Abgrenzungsansätzen. So unterscheidet bereits Castells (1977) urbane "Soziale Bewegungen" und Proteste nach ihrem revolutionären oder reformorientierten Potenzial. Künkel und Mayer (2012) greifen beispielsweise aus aktuellen städtischen Mobilisierungen solche heraus, die sich gegen den städtischen Neoliberalismus richten und Vollmer (2017) unterscheidet munizipalistische und rechtspopulistische Bewegungen entsprechend ihrer Zielsetzungen.

Innerhalb solch vielfältiger Unterscheidungsansätze wird hier ein empirisch begründetes analytisches Vorgehen gewählt, um Planungsproteste systematisch und vollständig zu erfassen. Entgegen den häufig dominierenden prozessualen Fragen - Welche Verfahren und Beteiligung? Zu welchem Zeitpunkt? - werden hierfür die Protest- bzw. Planungsinhalte herangezogen. Anders als im NIMBY-Diskurs werden sie allerdings aus den Selbstbeschreibungen der Protestierenden bestimmt. Die Untersuchung prozessualer Faktoren ist dann ein zweiter Schritt (vgl. Kapitel 5), der sich durch die vorangestellte Analyse der Inhalte auch nicht allein auf Möglichkeiten zur ,Verhinderung' von Planungsprotest begrenzen muss.

Ausgangspunkt ist das "strategische Framing", das seit Snow/Rochford/Worden et al. (1986; vgl. Kapitel 1.2) als ein wichtiges Protestmittel Eingang in die Protestforschung gefunden hat. Die von Protestakteuren diagnostizierten Probleme werden dabei als Anlässe, prognostizierte Lösungsvorschläge als Anliegen bezeichnet. Anders als in der planerischen Debatte spielen unter den 416 Fällen des Forschungsprojektes verfahrensbezogene Anlässe und Anliegen kaum eine Rolle (vgl. Abbildung 2). Nur sehr wenige Protestierende bemängeln hauptsächlich fehlende Beteiligung oder Verfahrensfehler. Meist beziehen sie sich in der Hauptsache auf materielle Konflikte in der gegenwärtigen räumlichen Situation oder als Ergebnis eines bestehenden Trends, einer vorgeschlagenen Planung oder eines angestrebten Bauvorhabens. Ihr wesentliches Ziel ist ein konkreter

4 NIMBY $=$ Not in my backyard. 


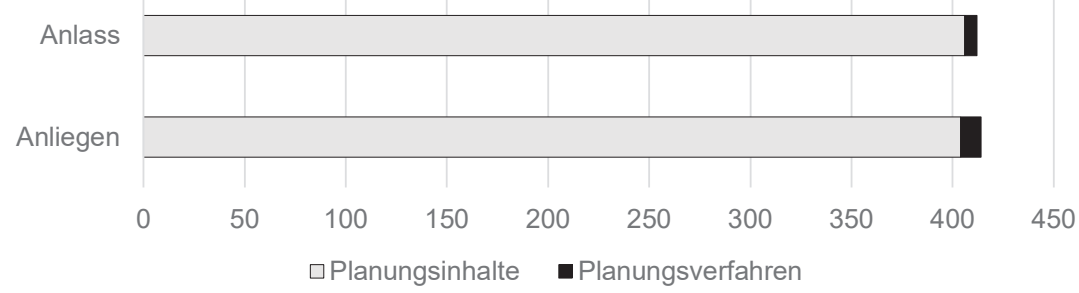

Abbildung 2: Anteil der Planungsinhalte und Planungsverfahren als Anlässe und Anliegen von Planungsprotesten in Berlin zwischen 2005 und $2015(\mathrm{~N}=416)$

Lösungsvorschlag zu einem Stadtentwicklungsproblem, eine Alternative zu bestehenden Plänen. In anderen Fällen besteht das Anliegen gerade darin, als negativ wahrgenommene Situationen oder ,ungeplante' Entwicklungen als Planungsaufgabe zu erkennen.

\subsection{Planungsproteste als alternative Problemlösungsansätze: eine planungsbezogene Typologie von Protestarten}

Betrachtet man das Gesamtspektrum der erfassten Proteste überwiegt zunächst der Eindruck der Vielfalt. Einzelne Planungsproteste können danach unterschieden werden, ob sich Betroffene oder Nichtbetroffene beteiligen (vgl. Kapitel 5.3), welche Planungsfelder und Planungsinstrumente angesprochen werden und welche Organisationsform die Protestnetzwerke ausbilden. Die hier vorgenommene Fokussierung auf Problemlösungsansätze stellt insofern eine deutliche Reduktion der Komplexität dar. Zugleich zeigt sich bereits eine hohe Diversität.

Im Gegensatz zu überlokalen Protesten und sozialen Bewegungen sind Anlässe und Anliegen von Planungsprotest in der Regel konkret. Protestierende benennen beispielsweise ein bestimmtes Planungsdokument oder die vorgesehene Zerstörung eines historischen Gebäudes als Anlass und fordern, dies zu verhindern, abzuschwächen oder zu verändern. In einigen Fällen schlagen sie auch konkrete alternative Lösungen, manchmal sogar eigene Pläne vor. Damit ist es im Planungskontext möglich, die ,doppelte Signatur' aus Anlass und Anliegen als alternativen Problemlösungsansatz aufzufassen. Protestierende greifen dabei oft unberücksichtigte Alternativen aus offiziellen Planungsdokumenten auf. Im Fall der Berliner Kleingärten forderten sie etwa, einen beschlossenen Bebauungsplanentwurf zur Rechtskraft zu bringen, was das Bezirksamt verweigerte. Das Beispiel zeigt, dass die Problemlösungsansätze konflikthaft sind und widersprüchliche Ansätze für Gegenwart und Zukunft der gebauten Umwelt beschreiben. Es bedeutet aber auch, dass Protestforderungen häufig weder unangemessen noch unerfüllbar sind.

Obwohl Anlässe und Anliegen dem strategischen Framing der Protestierenden entstammen, lassen sie sich auch als Basis für eine analytische Unterscheidung von Planungsprotesten nutzen. Da sich die zumeist konkreten Lösungsansätze stark auf die lokale Planung beziehen, ist eine auf innen aufbauende Typologie grundsätzlich bedeutsam. Dennoch ist der Erklärungsansatz beschränkt auf Zeit und Ort der empirischen Basis. Die in Tabelle 1 gezeigten und im Folgenden beschriebenen neun Protestarten sind für Berlin zwischen 2005 und 2015 gültig. Die analytisch gebildeten Kategorien erscheinen aber prinzipiell übertragbar. Insofern wird Berlin als empirische Grundlage im Folgenden vor allem als Illustration der grundsätzlichen analytischen Unterscheidungen verwendet.

Um zu einer Kategorisierung der Protestarten zu gelangen, werden die vielfältigen Anlässe und Anliegen jeweils anhand stark vereinfachter Kriterien unterschieden (in Tabelle 1 durch Linien voneinander getrennt). Anlässe werden danach unterschieden, ob sie sich auf bestehende Situationen im Stadtraum beziehen (situationsbezogene Anlässe) oder ein Planungsvorhaben den Ausgangspunkt bildet (planungsabhängige Anlässe). Diese einfache Differenzierung zeigt zunächst, ob Protestierende eine Veränderung der gebauten Umwelt einschließlich ihrer ,ungeplanten' Dynamiken wünschen oder ob die lokale räumliche Planung eine Veränderung vorsieht und damit den Protest verursacht. Die Anliegen werden in die drei Kategorien Ablehnung, Änderungen und Eigeninitiativen eingeteilt. Während die genaue Form der Ablehnung vom Anlass abhängt (vgl. nachfolgend unter „Planungsabhängige Planungsproteste“), 
Tabelle 1: Schematische Aufteilung der Arten aktueller planungsbezogener Proteste in Berlin

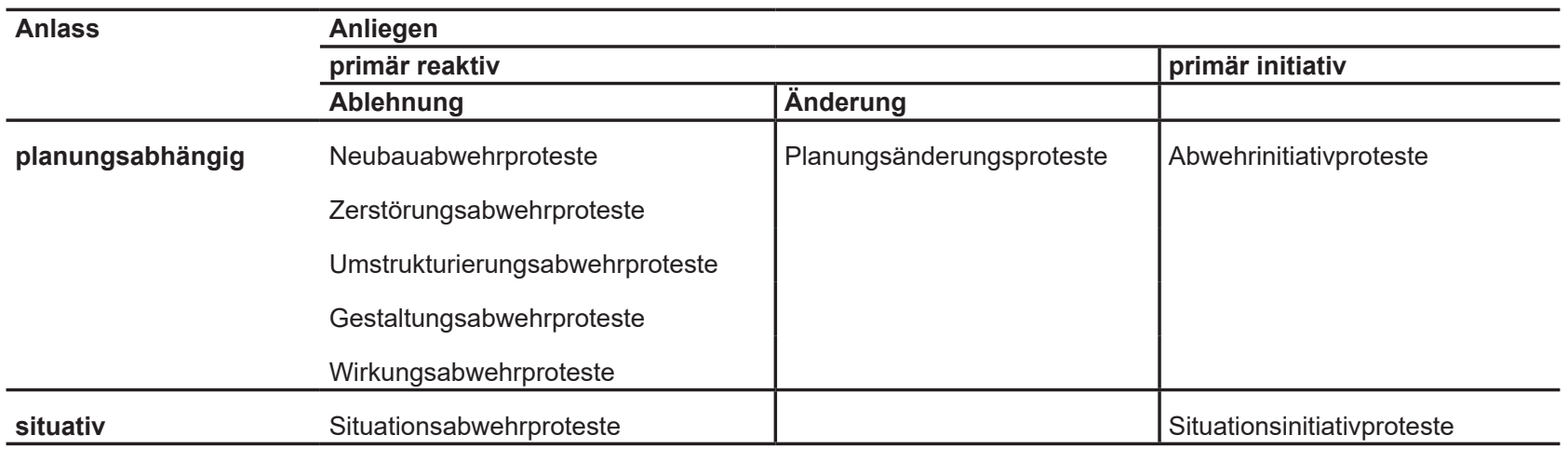

Tabelle 2: Anteile der Planungsprotestarten in Berlin zwischen 2005 und 2015 ( $N=416)$

\begin{tabular}{lll|l}
\hline Protestart & \multicolumn{2}{l}{ Anteil } & \\
\hline planungsabhängig & Neubauabwehrproteste & $16 \%$ & \multicolumn{2}{l}{ zusammen } \\
& Zerstörungsabwehrproteste & $9 \%$ & (Planungsabwehrproteste) \\
& Umstrukturierungsabwehrproteste & $7 \%$ & $9 \%$ \\
& Gestaltungsabwehrproteste & $12 \%$ & \\
& Wirkungsabwehrproteste & $12 \%$ & \\
\cline { 2 - 3 } & Planungsänderungsproteste & $7 \%$ & $17 \%$ \\
\hline situativ & Abwehrinitiativproteste & $10 \%$ & \\
\hline
\end{tabular}

wollen Änderungsproteste Planungen nicht gänzlich verhindern, sondern abwandeln. Eine sehr deutliche Abgrenzung $\mathrm{zu}$ ablehnenden Protesten findet sich in einer der beiden Fallstudien: Die Bürgerinitiative formulierte hier als Motto: „[Schnellstraße] ja, aber nicht so!“. Eine dritte Kategorie umfasst sämtliche Forderungen, die eigene (Planungs-)Ideen der Protestierenden in den Vordergrund stellen. Wo Bürgerinnen und Bürger initiativ werden und nicht (nur) reaktiv bleiben, wird für viele der positive Aspekt von Protest am deutlichsten.

Tabelle 2 zeigt, dass mehr als die Hälfte der erfassten Proteste Planungsabwehrproteste sind. Entsprechend der abgelehnten Planungsinhalte wurden hier fünf Protestarten unterschieden. Die nachfolgende Beschreibung gliedert sich anhand der prinzipiellen Unterscheidung in situationsbezogene und planungsabhängige sowie reaktive und initiative Planungsproteste. Da reaktive Proteste die Mehrheit der situationsbezogenen und planungsabhängigen Proteste darstellen und ohnehin dem gängigen Protestverständnis am nächsten kommen, ist eine eigenständige Erläuterung entbehrlich.

\section{Situationsbezogene Planungsproteste}

Der wesentliche Anlass von mehr als einem Viertel der dokumentierten Proteste ist nicht ein Planungsprozess, sondern eine bestehende Situation (27\%). Letztere stellt sich für die Protestierenden dabei durchaus unterschiedlich dar. Situationsbezogene Planungsproteste zeigen die kleinen und großen Probleme, die Bürgerinnen und Bürger in der gebauten Umwelt wahrnehmen. Gleichzeitig bleibt die geforderte planerische Lösung dieser Probleme häufig unscharf.

Gentrifizierung und andere Formen städtischer Restrukturierung zählen zu den häufigsten Anlässen von Situationsabwehrprotesten. Bemerkenswert ist, dass diese auch im Stadtentwicklungsdiskurs der vergangenen Jahre zentralen Themen durch die Protestakteure eben nicht direkt mit Planungen in Verbindung gebracht, sondern als allgemeine Trends dargestellt werden. Situationsinitiativproteste sind hingegen in der Regel kleinteilig und formulieren ,einfache' Lösungen für das direkte Lebensumfeld. Ein Schwerpunkt ist der Straßenverkehr - Lösungen reichen von Tempolimits über Ampeln bis hin zu Fußgängerüberwegen. 
Tabelle 3: Unterschiede planungsabhängiger Planungsproteste in Berlin 2005 bis 2015 anhand der häufigsten Ausprägungen ausgewählter Merkmale $(\mathrm{N}=416)$

\begin{tabular}{|c|c|c|c|c|}
\hline \multirow[t]{2}{*}{ Merkmal } & \multicolumn{4}{|c|}{ Art des planungsabhängigen Planungsprotests } \\
\hline & Neubau & Zerstörung & Umstrukturierung & Gestaltung \\
\hline Häufigster Anlass & Abwehr (58 \%) & Erhalt $(76 \%)$ & Erhalt $(72 \%)$ & Abwehr $(68 \%)$ \\
\hline Häufigstes Planungsfeld & Wohnen (44\%) & soziale Infrastruktur (40 \%) & Wohnen (56 \%) & $\begin{array}{l}\text { Stadterneuerung } \\
(34 \%)\end{array}$ \\
\hline $\begin{array}{l}\text { Häufigstes } \\
\text { Planungsinstrument }\end{array}$ & Bauleitplanung (38 \%) & $\begin{array}{l}\text { sonstige öffentliche } \\
\text { Instrumente (44 \%) }\end{array}$ & Bauleitplanung (46 \%) & $\begin{array}{l}\text { private Instrumente } \\
(42 \%)\end{array}$ \\
\hline $\begin{array}{l}\text { Häufigste verantwortliche } \\
\text { Verwaltungsebene }\end{array}$ & Gesamtstadt (51\%) & Bezirk (62 \%) & Bezirk (78 \%) & Bezirk $(75 \%)$ \\
\hline $\begin{array}{l}\text { Anteil Betroffenen- } \\
\text { proteste }\end{array}$ & $53 \%$ & $71 \%$ & $91 \%$ & $77 \%$ \\
\hline Durchschnittlich Beteiligte & 32.000 & 14.000 & 18.000 & 4.500 \\
\hline
\end{tabular}

\section{Planungsabhängige Planungsproteste}

Werden planungsabhängige Proteste betrachtet, die sich auf ein laufendes Planungsverfahren beziehen, so zeigt sich, welche inhaltlichen Probleme Bürgerinnen und Bürger in der lokalen räumlichen Planung sehen und welche Lösungen sie vorschlagen. Hier können Ablehnung, Änderung und Initiative als modellhafte Verkürzung eines kontinuierlichen Spektrums verschiedener Anliegen zwischen den drei Polen blanke Abwehr, Detailveränderung und unabhängiger Gegenvorschlag erachtet werden.

Ablehnung bedeutet, dass der soziale Wandel als Anliegen politischen Protests nur implizit artikuliert wird oder einer Umkehrlogik folgt: Protestierende Bürgerinnen und Bürger lehnen den sozialen Wandel ab, den Planung oder gegenwärtige Entwicklungstrends bringen, und wünschen - positiv formuliert - den Erhalt der bestehenden Situation. Insofern ist es häufig eine reine Frage des Framings, ob ein Protest sich gegen die Zerstörung oder Veränderung des Bestandes oder für dessen Erhalt einsetzt. Wie oben ausgeführt, wurden diese Proteste in der Typologie anhand der spezifischen Anlässe bzw. Planungsinhalte differenziert. Tabelle 3 zeigt, wie stark sich diese fünf Protestarten unterscheiden: Einige betonen die Abwehr, andere hingegen den Erhalt. Auslöser sind die öffentliche Bauleitplanung oder private Instrumente bzw. Bautätigkeit. Auffällig ist auch, dass Betroffene insbesondere bei Umstrukturierungsabwehrprotesten auftreten, während gegen Neubauvorhaben - beispielsweise bei neuen Stadtquartieren auch aus Mangel an direkter Betroffenheit - im Protest vor allem Gemeinwohlinteressen artikuliert werden.
Eine zusätzliche planungsabhängige Protestart wird nicht hinsichtlich der Anlässe unterschieden, sondern durch die vorgebrachten Anliegen. Dabei werden häufig detaillierte Planänderungen gefordert. In einigen Fällen geht es um die Minimierung von negativen Auswirkungen, in anderen um eine Abwandlung von Entwurf, Gestaltung oder Nutzung. Kann man die fünf übrigen planungsabhängigen Proteste klischeehaft auf ein ,Nein' zum vorgesehenen Plan reduzieren, so wird hier - wie im Fall der Ostberliner Schnellstraße - ein ,Ja, aber' geäußert. Eine siebte Art, das ,Nein, aber' der Abwehrinitiativen, wird nachfolgend beschrieben.

\section{Planungsproteste als Planungsinitiativen}

Ein knappes Viertel aller untersuchten Planungsproteste sind Initiativen, die Planung weniger als ,Gegner', sondern vor allem als Adressatin einer Forderung ansehen: „Gestaltet unseren Quartiersplatz!“, „Errichtet eine Ampel!“, „Baut einen Abenteuerspielplatz!“ Dies wird von Planenden häufig nicht als Protest wahrgenommen, sondern als (willkommenes) zivilgesellschaftliches Engagement. Dennoch bestehen Ähnlichkeiten mit den übrigen Protestarten, zunächst und vor allem beim (teilweise impliziten) Widerspruch zur offiziellen Planung, die häufig als Nicht-Planung wahrgenommen wird. Auch werden Forderungen nicht offen formuliert und in der Regel findet eine Festlegung auf einen bestimmten Lösungsansatz statt. Schließlich muss der positive Anspruch an Planung nicht dazu führen, dass der Widerspruch weniger deutlich wäre.

Letzteres gilt insbesondere für Abwehrinitiativproteste. Sie reagieren nicht auf eine Situation, sondern sind planungsabhängig. Ihre eigenständig vorgetragene 
Idee erwächst aus der Ablehnung einer bestehenden Planung. In Berlin bilden sie den kleineren Teil der Initiativproteste, doch wird in innen die ,doppelte Signatur' am deutlichsten: Anstelle des hegemonialen Problemlösungsansatzes entwickeln die Bürgerinnen und Bürger durch sie eine konkrete Alternative, etwa ein Nachbarschaftszentrum in einem Innenhof, der für eine Nachverdichtung mit Wohnnutzung vorgesehen ist, die Gründung einer Stiftung zur Errichtung neuer Wohngebäude statt eines Investors oder ein Kultur- anstelle eines Einkaufszentrums. Entsprechend besitzen sie stärkere Bezüge zur lokalen Planung als die oben beschriebenen situationsbezogenen Initiativen.

\subsection{Vielfalt von Protest jenseits der Typologie}

Im Gegensatz zu den in Kapitel 4.2 angeführten Unterscheidungen stellen die neun Protestarten eine Möglichkeit dar, das gesamte Protestspektrum überschneidungsfrei einzuteilen und dabei die fachliche, nicht aber die normative Perspektive der räumlichen Planung einzunehmen. Trotzdem zeigen sie nur einen Ausschnitt aus der aktuellen Vielfalt (vgl. Tabelle 4): Bürgerinnen und Bürger wollen in verschiedenen Bereichen mit vielfältigen Gründen und Zielen unterschiedlich an der Planung teilhaben. Die Protestierenden sind häufig, aber nicht immer betroffen, sie lassen sich nur selten eindeutig den überlokalen Sozialen Bewegungen zuordnen und nutzen verschiedene Aktionsformen, um auf ihre Anliegen aufmerksam zu machen. Die meisten Proteste werden auf Bezirksebene und von anlassbezogen gebildeten Bürgerinitiativen durchgeführt. Nicht zuletzt sind viele unterschiedliche Planungsfelder und Planungsinstrumente Gegenstand der Proteste.

Dies liegt zunächst daran, dass die lokale räumliche Planung selbst vielfältig ist. Die Bandbreite der Instrumente, Ziele und Eingriffstiefen führt zwangsläufig zu einer Diversität in den Anlässen und Anliegen von planungsbezogener politischer Partizipation. In einer individualisierten, zugleich aber vernetzten Gesellschaft ist davon auszugehen, dass sich das Protestspektrum diversifiziert, wie das etwa für die beteiligten Personengruppen seit Langem beobachtet wird (Rucht 2006: 205). Dennoch überraschen die unterschiedlichen Protestarten und die sich daraus ergebenden, zum Teil entgegengesetzten Bezugnahmen auf die Planung: Sie kann (Mit-)Auslöserin sein, aber auch als Problemlöserin angefragt werden. Protest kann auf Planung reagieren, sie aber auch initiieren. Ein breites Spektrum von ein- facher wie eindeutiger Ablehnung planerischer Eingriffe bis hin zu sehr konkreten, ausgewogenen Änderungswünschen ist möglich. Die Vielfalt zeigt allerdings auch, dass eine ernsthafte planerische Auseinandersetzung mit dem Phänomen ebenso vielfältige Reaktionen hervorrufen müsste.

\section{Differenzierte planerische Reaktionen auf vielfältige Proteste}

\subsection{Vielfältiger Protest fordert differenzierte Konfliktanalysen und Reaktionen heraus}

Für Planungspraktikerinnen/-praktiker oder Politikerinnen/Politiker, die erstaunt über die vielen Bürgerproteste sind, halten mehr als 40 Jahre Protestforschung eine recht eindeutige Erkenntnis bereit: „[p]eople do not risk their own skin or sacrifice their time to engage in contentious politics unless they have good reason to do so" (Tarrow 2011: $10 \mathrm{f}$.). Protest ist also als rationale, normale politische Strategie mit Erfolgschance anzusehen. Die empirischen Befunde aus Berlin machen deutlich, dass diese Normalität Eingang in die Planungswirklichkeit gefunden hat und Planungsprotest weder als Krisenphänomen noch als Ausdruck fehlgeleiteter Planung zu verstehen ist (Herkenrath 2011: 32). Er kann unterschiedlichen politischen Zielen wie der Mobilisierung oder Einflussnahme dienen (Gamson 1975). Im Berliner Fall handelt es sich dabei im Wesentlichen um eine inhaltliche Einflussnahme. Protest ist also Ausdruck eines veränderten Machtbegriffs, als Anwendung von „power to" anstatt von „power over" (Wrong 2009). Es erscheint einleuchtend, dass kaum jemand mit Protest nur Planungsprozesse, sondern meist auch Inhalte verändern möchte. Dies unterscheidet Planungsprotest von vielen überlokalen Protesten, Bewegungen und nicht zuletzt Revolutionen, bei denen auch immaterielle Rechte und demokratische Verfahren im Mittelpunkt stehen können.

Wenn Protest jedoch nur eine Ausdrucksform städtischer Politik unter vielen darstellt, wird es wesentlich einfacher, seine vielschichtigen Beziehungen zur räumlichen Planung zu untersuchen. Seine Normalität drückt sich auch in der großen Bandbreite von Anwendungen im Rahmen von Planungsverfahren durch viele Menschen mit ganz unterschiedlichen Vorstellungen aus (vgl. Rucht 2006). Es ist zu vermuten, dass unter den 
Tabelle 4: Übersicht über Anteile der wichtigsten Merkmalsausprägungen ( $N=416)$

\begin{tabular}{|c|c|c|c|c|}
\hline \multirow{8}{*}{ 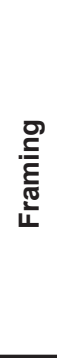 } & Anlass & Anliegen & Begründung & Betroffenheit \\
\hline & Vorhaben (57 \%) & Ablehnung (60 \%) & Bestand (36 \%) & ausschließlich Betroffene \\
\hline & Situation (19\%) & eigene Vorstellung (25\%) & Vorhaben (35 \%) & $(58 \%)$ \\
\hline & Wirkung (14\%) & Änderung (13\%) & Verfahren (4\%) & ausschließlich Nicht- \\
\hline & Trend $(8 \%)$ & Partizipation (2\%) & Grundsätze (2 \%) & Betroffene (21\%) \\
\hline & Verfahren (1\%) & & Protestumfang $(0,2 \%)$ & Betroffene und Nicht- \\
\hline & Bewegung $(0,2 \%)$ & & & Betroffene (12 \%) \\
\hline & keine Angaben (1\%) & keine Angaben (0,4\%) & keine Angaben (24\%) & keine Angaben (10\%) \\
\hline \multirow{13}{*}{ 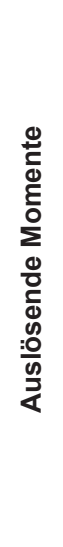 } & Planungsinstrument & Planungsfeld & Verfahrensstand & \\
\hline & sonstige öffentliche & soziale Infrastruktur (20 \%) & laufend (unspezifisch, & \\
\hline & Instrumente (22 \%) & Mobilität, technische Infrastruktur & $31 \%)$ & \\
\hline & private Instrumente (12 \%) & $(19 \%)$ & vorzeitig (26\%) & \\
\hline & Bauleitplanung (9 \%) & Stadterneuerung (18\%) & spät (18 \%) & \\
\hline & nachgeordnete Verfahren (5\%) & Wohnen (14 \%) & früh $(14 \%)$ & \\
\hline & Fachplanung (5\%) & Freiflächen (10 \%) & nachträglich $(6 \%)$ & \\
\hline & informelle Konzepte (2 \%) & Migration $(7 \%)$ & & \\
\hline & Beteiligung (2\%) & Gewerbe/Einzelhandel (5 \%) & & \\
\hline & & Neubau unspezifisch (2 \%) & & \\
\hline & & Immobilienwirtschaft (2 \%) & & \\
\hline & & Sonstiges (1\%) & & \\
\hline & keine Angaben (42 \%) & keine Angaben (1\%) & keine Angaben (12\%) & \\
\hline \multirow{13}{*}{ 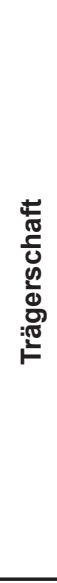 } & Organisationsform & Bewegungszugehörigkeit & Anzahl Beteiligte & Aktionsformen \\
\hline & anlassbezogene Vereinigung & keine Zugehörigkeit (66 \%) & zwischen 100 und 10.000 & demonstrative (21 \%) \\
\hline & $(77 \%)$ & stadtpolitischer Protestkomplex & $(25 \%)$ & Sammlungen (17 \%) \\
\hline & Einzelperson (8 \%) & $(18 \%)$ & unter $100(15 \%)$ & digitale $(17 \%)$ \\
\hline & Nichtregierungsorganisation & soziale Bewegung (16 \%) & über 10.000 (5 \%) & informative (15 \%) \\
\hline & $(7 \%)$ & & & expressive (5 \%) \\
\hline & Unternehmen (4 \%) & & & plakative (4 \%) \\
\hline & Sonstige (2\%) & & & Sonstiges (4 \%) \\
\hline & & & & Klage/Einspruch (4 \%) \\
\hline & & & & $\begin{array}{l}\text { Bürger-/Volksbegehren } \\
(3 \%)\end{array}$ \\
\hline & & & & direkte $(2 \%)$ \\
\hline & & & & direkte Ansprache (2 \%) \\
\hline & keine Angaben (3 \%) & & keine Angaben (55\%) & keine Angaben (9 \%) \\
\hline \multirow{9}{*}{ 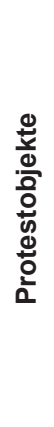 } & Objektart & Planungsebene & & \\
\hline & nur Verwaltung (32 \%) & Bezirk (54 \%) & & \\
\hline & Verwaltung und Politik (17 \%) & Gesamtstadt (41\%) & & \\
\hline & Verwaltung, Politik und & & & \\
\hline & Sonstige (11\%) & & & \\
\hline & nur Sonstige (10 \%) & & & \\
\hline & $\begin{array}{l}\text { Verwaltung und Sonstige (5 \%) } \\
\text { nur Politik (5\%) }\end{array}$ & & & \\
\hline & Politik und Sonstige (3 \%) & & & \\
\hline & keine Angaben (17\%) & keine Angaben (6 \%) & & \\
\hline
\end{tabular}




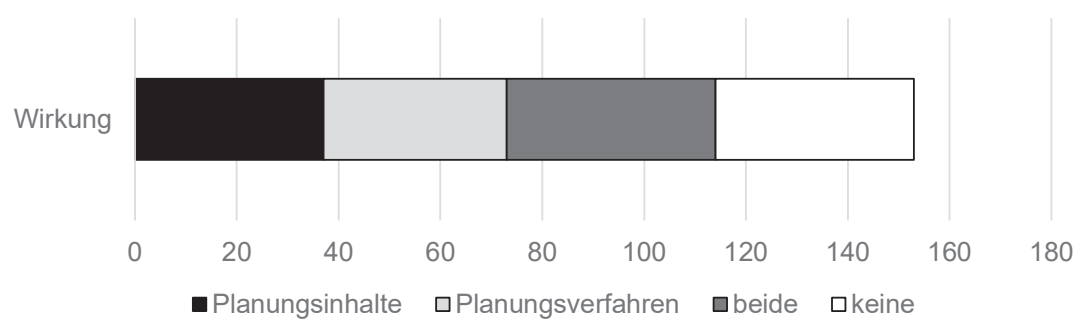

Abbildung 3: Anteil der veränderten Planungsinhalte und Planungsverfahren als Wirkung von Planungsprotesten in Berlin zwischen 2005 und $2015(\mathrm{~N}=416)$

,guten Gründen ' zu protestieren auch einige den gemeinwohlorientierten Zielen der Planung entsprechen. So lässt die große Zahl von Protestbeteiligten ohne direkte Betroffenheit darauf schließen, dass sich hinter dem vermeintlichen Eigennutz der Protestierenden auch soziale Verantwortung verbergen kann. Die vielfältigen Kombinationen von Anlässen und Zielen machen deutlich, dass Planungsproteste um alternative Ansätze und Verständnisse von einer gerechten Stadt ringen. Ein Blick darauf, worauf Aktivisten mit ihrer Politik abzielen (,what [...] activists [..] are tilting their politics at"; Mitchell/Attoh/ Staeheli 2015: 2646) sowie eine Untersuchung ihrer Lösungsvorschläge zeigt die politische Dimension ihres Handelns auf.

Die beschriebene Vielfalt legt nahe, mögliche Reaktionen der Planenden künftig systematischer zu betrachten. Proteste wurden bislang vielfach ignoriert oder allenfalls über kollaborative Prozesse eingebunden und entschärft (Othengrafen/Sondermann 2015: 14 ff.). In Abbildung 3 werden Fälle berücksichtigt, in denen Veränderungen durch Bürgerentscheide und ähnliche politische Entscheidungen außerhalb der Planung durchgesetzt wurden. Selbst wo Proteste als Teil eines pluralistischen politischen Alltags anerkannt werden, unterbleibt bislang eine weitergehende Analyse. Auch in der Planungspraxis lohnt es sich, genauer hinzusehen und die vielfältigen Anlässe und Ziele von Protest differenziert $z u$ betrachten, um angemessene und faire Reaktionen zu entwickeln. Die in den Protestarten ortsund zeitspezifisch ausformulierten Protestarten können hier einen Analyseansatz darstellen, der aber durch weitere Einordnungen ergänzt werden muss (vgl. Kapitel 5.3). In vielen Fällen kann ein gründlicheres Nachdenken zu verbesserten Problemlösungen und differenzierteren planerischen Antworten führen - und zwar nicht nur aus Sicht der Protestierenden. Im umgekehrten Sinn zwingen Protestforderungen, die aus planerischer Sicht individuellen Interessen deutlich den Vorrang gegenüber Gemeinwohlinteressen einräumen, zu ver- besserten Argumentationen der öffentlichen Hand. So können nicht unmittelbar aus dem Planungsvorhaben erkennbare Gemeinwohlinteressen oder weitergehende Entwicklungsüberlegungen besser im Kontext erläutert werden.

Eine schwierige Herausforderung ist die Integration von normal gewordenem Protest in die Planungsroutinen der Verwaltung. Meist bestehen weder Verfahrensregeln, die den Verwaltungen bestimmte Reaktionen vorgeben, noch übergeordnete Behörden, die nötigenfalls intervenieren. Doch auch wenn auf Protest durch aufwendige Beteiligungsverfahren reagiert wird, können diese nicht immer zu einer Befriedung beitragen. Eine Antwort darauf, wie "diese unregierbare Stadt" (Interview mit einem leitenden Berliner Senatsmitarbeiter im Herbst 2014) mit Instrumenten der Beteiligung und Konfliktbewältigung planerisch entwickelt werden sollte und welche Rolle dabei Proteste spielen, ist also offenbar bislang nicht in Sicht.

\subsection{Differenzierung der Reaktionen nach Protestarten}

Gleichwohl lassen sich erste Überlegungen zu einem Umgang mit Protesten in Anbetracht der vorgestellten Typologie anstellen. So können situationsbezogene Proteste politische Frühwarnsysteme ergänzen, wenn die Bevölkerung mit baulich-räumlichen Verhältnissen unzufrieden ist. Protest entsteht hier, weil nach Ansicht von Bürgerinnen und Bürgern die lokale räumliche Planung für eine Problemlösung zu kurz greift. Zwar können situationsbezogene Proteste weder unmittelbar in Planungsinitiativen umgesetzt werden, noch stellt die in innen geäußerte Unzufriedenheit immer ein zuverlässiges Stimmungsbild der Bevölkerung dar. Doch ein Monitoring situationsbezogener Proteste kann im Zusammenspiel mit anderen Mechanismen der Kommunikation wahrgenommener Missstände Stadtentwicklungspro- 
zesse qualifizieren. Neben traditionellen Formen, derartige Meinungsäußerungen zu kanalisieren und im Idealfall zu nutzen (etwa Petitionsrecht), wäre über eine Stärkung regulärer Feedback-Systeme nachzudenken. Dies könnte Bürgerinnen und Bürgern niedrigschwellig Möglichkeiten der Mitteilung eröffnen - ernsthaft, aber ohne direkte Hoffnung auf sofortige Veränderung. Inwiefern solche Angebote eine Alternative zur „unkonventionellen“ (Barnes/Kaase 1979: 151) Partizipation durch Protest darstellen und Möglichkeiten bieten, über den Mobilisierungs(miss)erfolg zu bemessen, wie breit die öffentliche Unterstützung ist (Tilly 2004: 3 f.), wäre gesondert zu diskutieren.

In besonderem Maße gilt dies für Situationsinitiativen, die eine bestimmte Planungsaktivität einfordern. Durch ihre Konkretheit und die oft ,einfachen' vorgeschlagenen Lösungen ist einerseits der Handlungsdruck höher, andererseits ein zeitnahes Aufgreifen durch Politik und Verwaltung möglich. Ignorieren scheint angesichts der immer wieder betonten Bedeutung zivilgesellschaftlichen Engagements für die Demokratie (vgl. die Beiträge in Becker/Gualini/Runkel et al. 2010) besonders problematisch. Eine sorgfältige planerische Prüfung und Abwägung der in der Regel laienhaften, interessengeleiteten Vorschläge scheint jedoch unerlässlich.

Reaktive Proteste dürften die größte Prominenz im Planungsalltag genießen. Durch die beschriebene innere Vielfalt zwischen Ablehnung, Änderung und Initiative sind aber gerade hier ein genauer Blick und Differenzierung notwendig. Gemeinsam ist den reaktiven Protesten, dass die von innen vorgeschlagenen Lösungsansätze Alternativen bereithalten. Hinsichtlich der Problembeschreibungen sind hingegen von Fall zu Fall Unterschiede erkennbar. Viele Protestakteure werden der planerischen Problembeschreibung zumindest teilweise zustimmen, gegebenenfalls aber bestimmte einzelne Aspekte anders bewerten. Nur selten stimmen die Auffassungen hier überhaupt nicht überein. Im Gegensatz zu situationsbezogenen Protesten löst das Planungshandeln die reaktiven Proteste mit aus, und damit werden die Planenden zumindest von den Protestierenden häufig als Konfliktpartei wahrgenommen, der zugleich die Verfahrenshoheit und die Rolle des Mittlers zukommt.

Angesichts der Angst, durch Planungsabwehrproteste in den Möglichkeiten einer Steuerung der räumlichen Entwicklung behindert zu werden, erscheint es nachgerade unerlässlich, sich ihre Normalität zu vergegenwärtigen. „Stuttgart 21" und das „Tempelhofer Feld“ in Berlin zeigen, dass es sich lohnt, frühzeitig mit ihnen zu rechnen und die in innen vertretenen Argumente sorgfältig aufzugreifen. Die Vehemenz, mit denen sie bisweilen vorgetragen werden, erfordert eine angemessene Behandlung und Abwägung der Argumente sowie eine sachliche Kommunikation. Nur so kann auch im Falle einer Zurückweisung sichergestellt werden, dass keine grundsätzliche Vernachlässigung der dahintersteckenden Belange vermutet wird. In diesem Zuge ist insbesondere eine Kontextualisierung erforderlich, die über den gerade verhandelten Einzelfall hinaus aufzeigt, wo und wann diese Belange ,zu ihrem Recht' kommen. Die Schwierigkeit der Reaktion auf diese in Berlin besonders häufigen Proteste liegt darin, dass die Differenz zwischen offiziellem und alternativem Lösungsansatz am größten ist und es nicht selten zu Anfeindungen von Entscheidungsträgern/-trägerinnen, gegebenenfalls auch Planerinnen/Planern, kommt. Letztere sind aber auch dafür verantwortlich, nicht einfach den am lautesten vorgetragenen Argumenten zu folgen, sondern im Sinne des Gemeinwohls Belange Dritter hinreichend zu berücksichtigen. Planungsabwehrinitiativen verkomplizieren diese Situation, wenn sie Gegenvorschläge zur aktuellen Planung unterbreiten, die sorgfältig argumentativ abgewogen werden müssen. Der alternative Problemlösungsansatz ermöglicht dann auch eine öffentliche Diskussion um Vorzüge und Nachteile beider Varianten.

Angesichts des hohen Konfliktpotenzials reaktiver Proteste ist es wichtig, Planungsänderungsproteste zu erkennen. Hier ist der materielle ,Streitwert' geringer, eine Berücksichtigung innerhalb der Planung wird in der Regel einfacher und der Vorschlag der Protestakteure zugleich weiter ausgearbeitet und ausführlicher begründet sein. Die empirischen Befunde zeigen, dass hier nicht selten auch fachliche Kompetenzen bestehen. Dies ermöglicht eine Kommunikation, die auf die vorhandene Expertise eingeht. Freilich muss es nicht zwangsläufig dazu führen, dass sich die Protestierenden in der Abwägung aller Interessen durchsetzen. Das oben genannte Fallbeispiel der Schnellstraße zeigt, dass auch Akteure in Planungsänderungsprotesten zunächst von der Neutralität der Planenden überzeugt werden wollen, bevor eine erfolgreiche Kommunikation möglich wird, mit der beispielsweise Klagen vorgebeugt werden kann. Zugleich zeigt sich, dass Planungsänderungen einer genauen Auseinandersetzung mit den Unterschieden zwischen offiziellem und alternativem Problemlösungsansatz bedürfen: Wurde die Schnellstraße in der Planung als Lösung für eine Reihe von Problemen betrachtet, galt sie den Betroffenen primär als Lösung für die starke Belastung anderer Verkehrswege. Daraus resultierten deutliche Unterschiede in der Bewertung einzelner Lösungsvarianten. Das dort gewählte Partizipati- 
onsangebot eines Planungsbeirats ermöglichte zudem, die erweiterte Teilhabe nicht auf die besonders aktiven, kompetenten oder ,lauten' Akteure zu beschränken.

\subsection{Alternative Differenzierung der Reaktionen nach Betroffenheit}

Wie in Kapitel 4.1 gezeigt, ist das Spektrum des Planungsprotests deutlich differenzierter, als die Protestarten dies abbilden können. Daher soll hier beispielhaft eine ergänzende Differenzierung nach der Betroffenheit angeführt werden. Gerade in der Kritik an NIMBYs und „Wutbürgern“, aber etwa auch bei Othengrafen und Sondermann (2015: 9), wird häufig von Betroffenheit als alleiniger Protestmotivation ausgegangen, während die politisch-strategische Protestauffassung seit Gamson (1975) gerade von vielfältigen Protestzielen und damit auch Teilnahmemotivationen ausgeht (vgl. Kapitel 2). So lassen sich auch Planungsproteste danach unterscheiden, ob sie allein von Personen organisiert werden, deren Interessen direkt von der Planung berührt werden, oder ob sich (auch) Personen daran beteiligen, die sich stellvertretend für Dritte oder für Gemeinwohlinteressen engagieren. Die Analyse der Falldaten zeigt, dass für gut ein Fünftel aller untersuchten Protestfälle keine Beteiligung Betroffener erkennbar war $(21 \%)$ und in weiteren $12 \%$ Betroffene und Nichtbetroffene gemeinsam protestierten. Die planungsabhängigen Protestarten in Tabelle 3 verdeutlichen exemplarisch, wie sehr diese Anteile schwanken.

Planungstheoretisch wie juristisch folgt daraus zunächst die Frage der Legitimität (vgl. Maier 2011; Behringer 2015). In einigen Verfahrenstypen wird formal Betroffenen eine höhere Berechtigung zugesprochen, sich für ihre Interessen einzusetzen. Für Unbeteiligte kann im Grundsatz davon ausgegangen werden, dass sie sich stärker für Gemeinwohlbelange einsetzen. Planung muss beiden einen angemessenen Rang zubilligen. Werden berechtigte Interessen von Betroffenen über Gebühr in Frage gestellt, liegt im Extremfall ein Abwägungsfehler vor. Nicht immer stimmen die gesetzlichen Regeln zur Betroffenheit mit der Einschätzung vor Ort überein, etwa wenn Kleingärtnerinnen/Kleingärtner als Unterpächterinnen/-pächter im Baugenehmigungsverfahren nicht beteiligt werden müssen. Protesthandeln kann insofern darauf hindeuten, dass Betroffene das Gebot der Fairness in diesem Zusammenhang als verletzt ansehen. Eher Unbeteiligte wiederum signalisieren mit ihrem Protest, den sie trotz fehlenden Eigeninteresses artikulieren, dass Planungsentscheidungen außer- halb der fachlichen Binnenperspektive ganz anders bewertet werden. Der mögliche Einwand, dass dafür ein Mangel an Informationen, eine Überbewertung einzelner Belange oder eine kurzsichtige Einschätzung langfristiger Entwicklungsnotwendigkeit ausschlaggebend seien und man auf derartige Aktionen allenfalls mit verbesserter Information reagieren müsse, ist zu pauschal. Allein der Aufwand, mit dem Protest nicht nur initiert, mobilisiert und aufrechterhalten, sondern mit ausgefeilten Argumenten unterstützt wird, steht hierzu im Widerspruch. Hinzu kommt nicht selten eine nachgewiesene Kompetenz zumindest für die vorgetragenen Belange etwa im Naturschutz - und die planungsgeschichtliche Erfahrung mit den heute häufig als berechtigt bewerteten Planungsprotesten früherer Jahrzehnte (,Kahlschlagsanierung"; Bodenschatz/Heise/Korfmacher 1983). Protest kann hier mindestens als Hinweis darauf verstanden werden, dass planerische Bewertungen ganz unterschiedlich ausfallen können, selbst wenn man sich sehr gründlich mit einem Planungsfall auseinandersetzt, und dass hierüber nicht ausführlich genug diskutiert wurde. Nicht zuletzt das Fallbeispiel der Westberliner Kleingärten, deren ökologische und klimatische Bedeutung jahrzehntelang durch offizielle Planungsdokumente bestätigt wurde, zeigt auch, dass Lösungsalternativen nicht nur zwischen Planungs- und Protestakteuren umstritten sind, sondern auch innerhalb der Planung selbst. Entsprechend braucht es stets einen politischen Aushandlungs- und Entscheidungsprozess, der über diese Alternativen informiert ist - notfalls durch Protest.

\section{Von der Akzeptanz der Normalität zur differenzierten Konfliktbearbeitung - Fazit}

Der Beitrag zeigt beispielhaft, wie es möglich ist, Planungsproteste in ihrer Vielfalt zu erfassen und eine Unterscheidung vorzunehmen. Daraus resultiert eine vorläufige Typologie, die aus der Vielfalt der Protestereignisse in Berlin entwickelt wurde. Die grundsätzliche Gegenüberstellung von situationsbezogenen und planungsabhängigen Protestanlässen sowie primär ablehnenden, auf Änderungen abzielenden und initiativen Anliegen ist dabei auf andere Orte übertragbar - ob die systematisierende Typologie angesichts des jeweiligen lokalen Protestspektrums ebenfalls über den jeweiligen Ort hinaus Gültigkeit reklamieren kann, bedarf der Überprüfung. Dies wird einerseits am Fehlen verfahrensbezogener Anlässe und Anliegen in der Typologie deut- 
lich: Wären sie andernorts häufiger vertreten, müssten entsprechende Typen ergänzt bzw. bestehende ersetzt werden. Andererseits zeigt die innere Differenzierung der planungsabhängigen Proteste, dass auch hier empirisch bestimmte Häufigkeiten in die analytische Typisierung einbezogen wurden und zu einem entsprechend ortsspezifischen Ergebnis führen.

In Kapitel 5 wurde am Merkmal der Betroffenheit zudem gezeigt, dass differenzierte Analysen und Reaktionen nicht allein durch die dargelegten Protestarten möglich sind. Auch in Berlin fehlen sie heute noch weitgehend. Dennoch wird hier - manchmal zwangsläufig das Auftreten von Protesten längst als Normalität hingenommen und als nicht ungewöhnlich in einer Demokratie angesehen. Wenngleich belastbare empirische Daten bislang fehlen, spricht einiges dafür, dass dies zumindest als Tendenz auch auf andere Städte und Regionen der Bundesrepublik zutrifft. Mit der beobachtbaren Digitalisierung auch von Protestformen (Sassen 2012; Butler 2015) und den vielfältigen Beteiligungsangeboten in Zeiten der „partizipatorischen Revolution“ (Kaase 1982) gibt es heute wesentlich mehr Möglichkeiten einer Planungsbeteiligung und man kann die hierzu genutzten Instrumente aus einem breiten Spektrum wählen, das deutlich über die formalisierten Formen hinausreicht.

Verallgemeinerbar ist aber in jedem Fall der Paradigmenwechsel, Planungsprotest als normal zu akzeptieren. Dies kann möglicherweise den Planungsalltag bereichern und vermitteln, dass sich Beteiligung an Verfahren lohnt. Es soll aber gerade nicht als Argument für eine agonistische Perspektive auf Protest und Konflikt angesehen werden, aus der häufig entweder eine einseitig positive Bewertung von Protesten oder eine alleinige Feststellung der verschiedenen Positionen, aber kaum eine Möglichkeit zur Konfliktbearbeitung folgt (vgl. Othengrafen/Sondermann 2015: 15). Akzeptanz, Analyse und Differenzierung ermöglichen gerade eine Bearbeitung der in eigenständiger Partizipation vorgetragenen Konflikte innerhalb rechtsstaatlicher und demokratischer Verfahren.

Noch auszuloten ist, wie das Erpressungspotenzial, das in der Krawalligkeit von Protesten im Extremfall liegt, angemessen eingeschätzt werden kann. Für faire Planungsprozesse muss es nach wie vor darum gehen, dass die Kraft des Arguments zählt. Lautstärke, Unbequemheit, moralischer Impetus und Blockadedrohungen sollten keine legitimen Instrumente der Machtausübung sein - zumal dort, wo sich eine akzeptierte Protestpartizipation als erfolgversprechende Strategie für ohnehin mächtige Akteure darstellt. Deuten sie allerdings darauf hin, dass die Protestakteure deshalb zu innen greifen, weil sie sich selbst als machtlos und bedroht fühlen, dann gilt es, diese Symptome angemessen zu würdigen und den Blick für die Fairness von Planungsverfahren auf alle Akteure und deren Strategien zu lenken. Umgekehrt dürfen unsachgemäße oder ungemessen laute und unbequem vorgetragene Argumente auch nicht ignoriert oder geringer geschätzt werden als fachlich versierte.

Durch die Normalisierung von Protest als politischer Partizipation ist eine weitere Demokratisierung von Stadtentwicklungsprozessen vorstellbar. Doch so lange die beschriebene Normalisierung nur mit der Hinnahme von Protestformen verbunden ist, ohne den Umgang mit innen zu verändern, werden Planungsprozesse lediglich komplexer, weniger steuerbar und stärker den Einflüssen von Einzelinteressen ausgesetzt. Die einfache Hinnahme riskiert überdies, noch stärker als bisher den wenig Artikulationsfähigen in der Gesellschaft das angemessene Gehör zu verweigern. Eine weitere Demokratisierung wäre das Gegenteil, und daher steht eine aktive und vertiefte Auseinandersetzung mit Planungsprotest auf allen Ebenen von Planung und Politik an.

\section{Literatur}

Barnes, S. H.; Kaase, M. (1979): Political Action. Mass Participation in Five Western Democracies. Beverly Hills.

Becker, E.; Gualini, E.; Runkel, C.; Graf Strachwitz, R. (Hrsg.) (2010): Stadtentwicklung, Zivilgesellschaft und bürgerschaftliches Engagement. Stuttgart.

Behringer, J. (2015): Zur Wahrnehmung von Partizipation: Perspektiven und Legitimation. In: PND online. http://www. planung-neu-denken.de/content/view/319/41 (04.09.2019).

Bertram, G. (2013): Kritisiert die Kritiker_innen! Kommentar zu Margit Mayers „Urbane soziale Bewegungen in der neoliberalisierenden Stadt“. In: sublurban. Zeitschrift für kritische Stadtforschung 1, 1, 169-174.

Bertram, G. (2019): „Aber nicht so!“ Theorie und Praxis, Ansprüche und Wirklichkeiten bürgerschaftlichen Planungsprotests in Berlin 2005 bis 2015. Dissertation an der Universität Kassel. doi: 10.17170/kobra-20190129119 (04.09.2019).

Bertram, G. F.; Altrock, U. (2017): Kampf um die Städte? Protestpartizipation in der lokalen Demokratie. https://www. bpb.de/politik/innenpolitik/stadt-und-gesellschaft/216886/ protestpartizipation-in-der-lokalen-demokratie (04.09.2019).

Bodenschatz, H.; Heise, V.; Korfmacher, J. (1983): Schluss mit der Zerstörung? Stadterneuerung und städtische Opposition in West-Berlin, Amsterdam und London. Gießen. = WerkbundArchiv 11.

Brake, K. (2012): Berlin - relative Reurbanisierung einer gewendeten Stadt. In: Brake, K.; Herfert, G. (Hrsg.): Reurbanisierung. Materialität und Diskurs in Deutschland. Wiesbaden, 258-286. doi: 10.1007/978-3-531-94211-7

Butler, J. (2015): Notes toward a performative theory of assembly. Cambridge. 
Castells, M. (1977): The Urban Question. A Marxist Approach. London. $=$ Social Structure and Social Change 1.

Dohnke, J. (2014): Spreeufer für alle! Was bleibt von „Mediaspree versenken"? In: Holm, A. (Hrsg.): Reclaim Berlin. Soziale Kämpfe in der neoliberalen Stadt. Berlin, 316-342.

Eckert, R. (1970): Politische Partizipation und Bürgerinitiative. In: Boss-Stenner, H.; von Pufendorf, U.; Schade, K. F. (Hrsg.): Partizipation. Aspekte politischer Kultur. Opladen, 30-47. doi: 10.1007/978-3-663-02813-0

Fainstein, S. S.; Fainstein, N. I. (1985): Economic Restructuring and the Rise of Urban Social Movements. In: Urban Affairs Review 21, 2, 187-206. doi: 10.1177/004208168502100204

Gamson, W. A. (1975): The strategy of social protest. Homewood.

Goodwin, J.; Jasper, J. M. (2003): Introduction. In: Goodwin, J.; Jasper, J. M. (Hrsg.): The social Movements Reader. Malden, 1-7.

Guggenberger, B. (2009): Bürgerinitiativen. In: Andersen, U.; Woyke, W. (Hrsg.): Handwörterbuch des politischen Systems der Bundesrepublik Deutschland. Wiesbaden, 39-45.

Haughton, G.; Gilchrist, A.; Swyngedouw, E. (2016): "Rise Like Lions After Slumber": Dissent, Protest and (Post-)Politics in Manchester. In: Territory, Politics, Governance 4, 4, 472-491. doi: 10.1080/21622671.2016.1141705

Herkenrath, M. (2011): Die Globalisierung der sozialen Bewegungen. Transnationale Zivilgesellschaft und die Suche nach einer gerechten Weltordnung. Wiesbaden. doi: 10.1007/978-3-53193118-0

Holm, A.; Kuhn, A. (2010): Häuserkampf und Stadterneuerung. In: Blätter für deutsche und internationale Politik 45, 3, 107-115.

Kaase, M. W. (1982): Partizipatorische Revolution: Ende der Parteien? In: Raschke, J. (Hrsg.): Bürger und Parteien. Ansichten und Analysen einer schwierigen Beziehung. Bonn, 173-189.

Krau, I. (2011): Stuttgart 21 und Verfahrensfragen. In: PND online 2. http://www.planung-neu-denken.de/texte-mainmenu-41/197kraus212-2011 (04.09.2019).

Künkel, J.; Mayer, M. (Hrsg.) (2012): Neoliberal Urbanism and its Contestations. Crossing Theoretical Boundaries. Basingstoke.

Lake, R. W. (1993): Planners' Alchemy Transforming NIMBY to YIMBY. Rethinking NIMBY. In: Journal of the American Planning Association 59, 1, 87-93. doi: 10.1080/01944369308975847

Lennon, M. (2017): What is planning? In: The Town Planning Review 88, 2, 147-151. doi: 10.3828/tpr2017.11

Lindner, W. (1996): Jugendprotest seit den fünfziger Jahren. Dissens und kultureller Eigensinn. Opladen. = Studien zur Jugendforschung 17.

Lopes de Souza, M. (2006): Social movements as 'critical urban planning' agents. In: City 10, 3, 327-342. doi: 10.1080/13604810600982347

Maier, W. (2011): Demokratische Legitimation im Wandel. In: Kommune. Forum für Politik, Ökonomie und Kultur 18, 6, 6-10.

Mayer, M. (2008): Städtische soziale Bewegungen. In: Roth, R.; Rucht, D. (Hrsg.): Die sozialen Bewegungen in Deutschland seit 1945. Ein Handbuch. Frankfurt am Main, 293-318.

Melucci, A. (1985): The Symbolic Challenge of Contemporary Movements. In: Social Research 52, 4, 789-816.

Menzl, M. (2014): Nimby-Proteste. Ausdruck neu erwachten Partizipationsinteresses oder eines zerfallenden Gemeinwesens? In: Gestring, N.; Ruhne, R.; Wehrheim, J.
(Hrsg.): Stadt und soziale Bewegungen. Wiesbaden, 65-81. doi: 10.1007/978-3-658-01398-1

Mitchell, D.; Attoh, K.; Staeheli, L. (2015): Whose city? What politics? Contentious and non-contentious spaces on Colorado's Front Range. In: Urban Studies 52, 14, 2633-2648. doi: 10.1177/0042098014550460

Othengrafen, F.; Sondermann, M. (2015): Konflikte, Proteste, Initiativen und die Kultur der Planung - Stadtentwicklung unter demokratischen Vorzeichen. In: Othengrafen, F.; Sondermann, M. (Hrsg.): Städtische Planungskulturen im Spiegel von Protesten, Konflikten und Initiativen. Berlin, 7-30. = Reihe Planungsrundschau 23.

Roth, R.; Rucht, D. (2008): Einleitung. In: Roth, R.; Rucht, D. (Hrsg.): Die sozialen Bewegungen in Deutschland seit 1945. Ein Handbuch. Frankfurt am Main, 9-36.

Rucht, D. (2001): Protest und Protestereignisanalyse: Einleitende Bemerkungen. In: Rucht, D. (Hrsg.): Protest in der Bundesrepublik. Strukturen und Entwicklungen. Frankfurt am Main, 7-25.

Rucht, D. (2006): Politischer Protest in der Bundesrepublik Deutschland: Entwicklungen und Einflussfaktoren. In: Hoecker, B. (Hrsg.): Politische Partizipation zwischen Konvention und Protest. Eine studienorientierte Einführung. Opladen, 184-208.

Rucht, D.; Ohlemacher, T. (1992): Protest Event Data: Collections, Uses and Perspectives. In: Diani, M.; Eyerman, R. (Hrsg.): Studying collective action. London, 76-106.

Sassen, S. (2012): Saskia Sassen über die Macht der Machtlosen. In: Der Standard vom 25. Februar 2012, A3.

Schiller, T. (1970): Parteien und Bürgerinitiative. In: Boss-Stenner, H.; von Pufendorf, U.; Schade, K. F. (Hrsg.): Partizipation. Aspekte politischer Kultur. Opladen, 114-121. doi: 10.1007/9783-663-02813-0

Selle, K. (2011a): „Particitainment“ oder: Beteiligen wir uns zu Tode? In: PND online 3. http://www.planung-neu-denken.de/ images/stories/pnd/dokumente/3_2011/selle_particitainment. pdf (04.09.2019)

Selle, K. (2011b): Something went wrong. In: PND online 2. http://www. planung-neu-denken.de/images/stories/pnd/ dokumente/2_2011/s21_selle.pdf (04.09.2019).

Selle, K. (2013): Über Bürgerbeteiligung hinaus. Stadtentwicklung als Gemeinschaftsaufgabe? Detmold.

Snow, D. A.; Benford, R. D. (1988): Ideology, Frame Resonance, and Participant Mobilization. In: Klandermans, B.; Kriesi, H.; Tarrow, S. G. (Hrsg.): From Structure to Action. Comparing Social Movement Research Across Cultures. Greenwich, 197217.

Snow, D. A.; Rochford Jr., E. B.; Worden, S. K.; Benford, R. D. (1986): Frame Alignment Processes, Micromobilization, and Movement Participation. In: American Sociological Review 51, 4, 464-481. doi: 10.2307/2095581

Tarrow, S. (2011): Power in movement. Social movements and contentious politics. Cambridge.

Tilly, C. (2004): Social movements, 1768-2004. Boulder.

Vogelpohl, A.; Vollmer, L.; Vittu, E.; Brecht, N. (2017): Die Repolitisierung des Wohnens. Städtische soziale Bewegungen für ein Recht auf Wohnen und auf Stadt in Hamburg, Berlin, Jena und Leipzig. In: Schönig, B.; Kadi, J.; Schipper, S. (Hrsg.): Wohnraum für alle?! Perspektiven auf Planung, Politik und Architektur. Bielefeld, 105-130. 
Vollmer, L. (2017): Keine Angst vor Alternativen. Ein neuer Munizipalismus. Über den Kongress „FearlessCities“, Barcelona, 10./11. Juni 2017. In: sublurban. Zeitschrift für kritische Stadtforschung 5, 3, 147-156.

Walter, F. (2013): Bürger in Bewegung. Zur Einführung. In: Marg, S.; Geiges, L.; Butzlaff, F.; Walter, F. (Hrsg.): Die neue Macht der Bürger. Reinbek, 9-13.

Wrong, D. (2009): Power: Its forms, bases and uses. New Brunswick. 